\title{
El sida: explorando cómo se modelan las opiniones
}

\author{
Sophie Tazé, Universidad de Lille $1^{1}$
}

\section{Resumen}

En este trabajo se analiza la interdependencia de opiniones en relación con el SIDA. EI análisis empírico permite comprobar que la convergencia en díadas de confidentes y la estabilización de las opiniones en estructuras sociométricas cerradas.

Palabras clave: redes sociales - SIDA - opiniones.

\section{Abstract}

The interdependence of opinions regarding AIDS is examined. Both the convergence of opinions in dyads of confidents and the stabilization of the opinions in closure networks are observed.

Key words: social network - AIDS - opinions.

La epidemia del SIDA ha creado, desde principios de los años 80 , un cierto número de problemas y cuestiones de salud pública: ¿Cómo limitar la epidemia? ¿Cómo disminuir los riesgos de contaminación (por transfusión sanguínea y transmisión sexual)? ¿Cómo mejorar las condiciones de vida de los enfermos y, concretamente, contrarrestar la discriminación de la que son objeto? ¿Cómo informar sin alarmar las personas en situación de riesgo, así como la población general y en particular los jóvenes? El SIDA se ha constituido como un objeto de las ciencias sociales en torno a estas cuestiones dando lugar a importantes encuestas sobre el conocimiento, las creencias y también los comportamientos de los actores frente a la enfermedad y a los riesgos que conlleva.

Por otra parte, la introducción de los nuevos tratamientos en 1996, también llamados triterapias, ha modificado el contexto terapéutico: el sida ya no es percibido como una "maldición" sino como una "enfermedad con la que se puede vivir". Estos cambios de la percepción de la enfermedad y de su gravedad han llevado consigo un retorno de los comportamientos arriesgados, particularmente visibles en la generación "post-sida" - es decir, las personas que han comenzado su vida sexual después de las grandes campañas de prevención (15-25 años). Así pues, un joven de 19 años puede decir hoy en día: “¿El sida? Si, tengo miedo por mi hermano, pero yo, mientras me acueste con personas de mi edad, no tengo nada que temer".

\footnotetext{
${ }^{1}$ Este artículo ha sido traducido por Ainhoa de Federico, Universidad de Toulouse 2, a quien agradezco su gentileza. El trabajo forma originalmente parte del proyecto "El trabajo en red de los profesionales de la salud: conceptos y herramientas del análisis de redes sociales. Apoyo a la gestión de la red de formadores del Plan Integral de Tabaquismo de Andalucía », de la Escuela Andaluza de Salud Pública, coordinado por Isidro Maya Jariego.
} 
REDES- Revista hispana para el análisis de redes sociales

Vol. 18, \#1 Junio 2010

http://revista-redes. rediris.es

En este contexto de desdramatización se plantea una cuestión importante: ¿cómo se forman opiniones los individuos de un cierto número de aspectos de esta enfermedad?

Si de dichas opiniones se derivan los comportamientos de los actores frente al riesgo (lo que está por demostrar), entonces es crucial comprender porqué, por ejemplo, el joven del que hablamos dice no correr riesgos de contaminarse. Si consideramos que las opiniones no se producen en la cabeza de dicho actor aislado (postura individualista), ni que son vehiculadas del inconsciente colectivo hacia su conciencia individual (postura holista) ¿de dónde le viene entonces esta idea? ¿de los medios de comunicación? ¿de una intervención escolar de prevención? ¿de una charla con su hermano mayor? ¿de sus amigos? ¿de sus padres? Exploremos la siguiente posibilidad: las opiniones emergen y se forman durante las conversaciones (interaccionismo estructural).

Partiendo de la hipótesis que las opiniones se forman en las interacciones, nos podemos preguntar si:

a) las opiniones convergen más en ciertas relaciones. En ese caso ¿de qué relaciones se trataría? Dicho de otro modo, ¿no podríamos pensar que nuestro joven actor tenga tendencia a tener opiniones similares a las de alguno de sus interlocutores? ¿Daría esta convergencia una fuerza específica a la opinión compartida en esta relación?

b) Las opiniones son modeladas según las especificidades de la red de discusión en la que son producidas. Si algunos de su $\mathrm{s}$ interlocutores tienen opiniones diferentes, ¿se alineará el joven a una de ellas u oscilará de una a otra según las conversaciones? ¿Podríamos entonces identificar los procesos sociométricos implicados en los cambios de opinión de un actor, es decir, su versatilidad?

El estudio de los procesos a) de convergencia en la díada (relación formada entre dos individuos, b) de versatilidad en la triada (relación entre tres actores) puede llevarse a cabo según un protocolo de investigación específico consistente en registrar primeramente las opiniones producidas simultáneamente por un actor A y un actor B (cuestionario interactivo 1) y, posteriormente, las opiniones expresadas simultáneamente por el mismo actor $\mathrm{A}$ y un actor C (cuestionario interactivo 2 ).

Este estudio revela, en áreas de incertidumbre 1) el impacto de una característica relacional específica sobre la formación de opiniones convergentes cuando dos actores se expresan juntos respecto al sida y 2) una lógica estructural de estabilización de las opiniones cuando un actor se expresa sucesivamente con dos interlocutores diferentes sobre las mismas cuestiones. 
REDES- Revista hispana para el análisis de redes sociales

Vol. 18, \#1 Junio 2010

http://revista-redes. rediris.es

\section{La persistencia de la incertidumbre}

Un actor en nuestras sociedades puede encontrar difícil formarse cierta opinión sobre un problema a causa de la falta de información o, por el contrario, de su gran diversidad, o también por falta de confianza en dichos datos. Al abordar las cuestiones relativas al sida con estudiantes de sociología (edad media 19 años), se vuelve evidente que subsisten importantes zonas de incertidumbre frente a zonas de gran certeza respecto al SIDA.

Cuando se trata de ítems con un juicio como "hay que prohibir a las personas seropositivas el integrarse al sistema educativo", o con falsas creencias como "los nuevos tratamientos impiden la contaminación después de una relación sexual de riesgo", la homogeneidad de las respuestas traduce la existencia de zonas de representación de clara certeza. Sin embargo la incertidumbre persiste respecto a un gran número de cuestiones.

Hemos explorado la posibilidad de que existan interdependencias entre representaciones relativas a diferentes objetos como la prevención, la proximidad de la enfermedad, el miedo, la muerte o los nuevos tratamientos. ¿Piensa la gente que los nuevos tratamientos han provocado un cambio de los comportamientos preventivos? ¿Se tiene hoy en día menos miedo del sida? ¿Existe una relación entre le hecho de tener menos miedo y el de hablar más de la cuestión? Nuestra investigación ha buscado cuáles entre los diferentes temas muestran más incertidumbre y nos hemos interesado por las interdependencias que podrían ligar algunos de ellos.

El esquema presentado constituye un análisis susceptible de mostrar una estructura mínima de las interconexiones binarias entre las opiniones. Representa todas las relaciones estadísticas significativas que existen entre los ítems de dos en dos. La existencia de una relación significa, por ejemplo, "decir que tengo (o no) un deber de alertar a mis amigos sobre los riesgos de contaminación tiene algo que ver con el hecho de decir que se puede (o no) tener confianza en que las personas seropositivas informan a sus parejas".

Hemos identificado 20 correlaciones significativas entre todos los pares de ítems definiendo así una estructura tri-polar: 
REDES- Revista hispana para el análisis de redes sociales

Vol. 18, \#1 Junio 2010

http://revista-redes. rediris.es

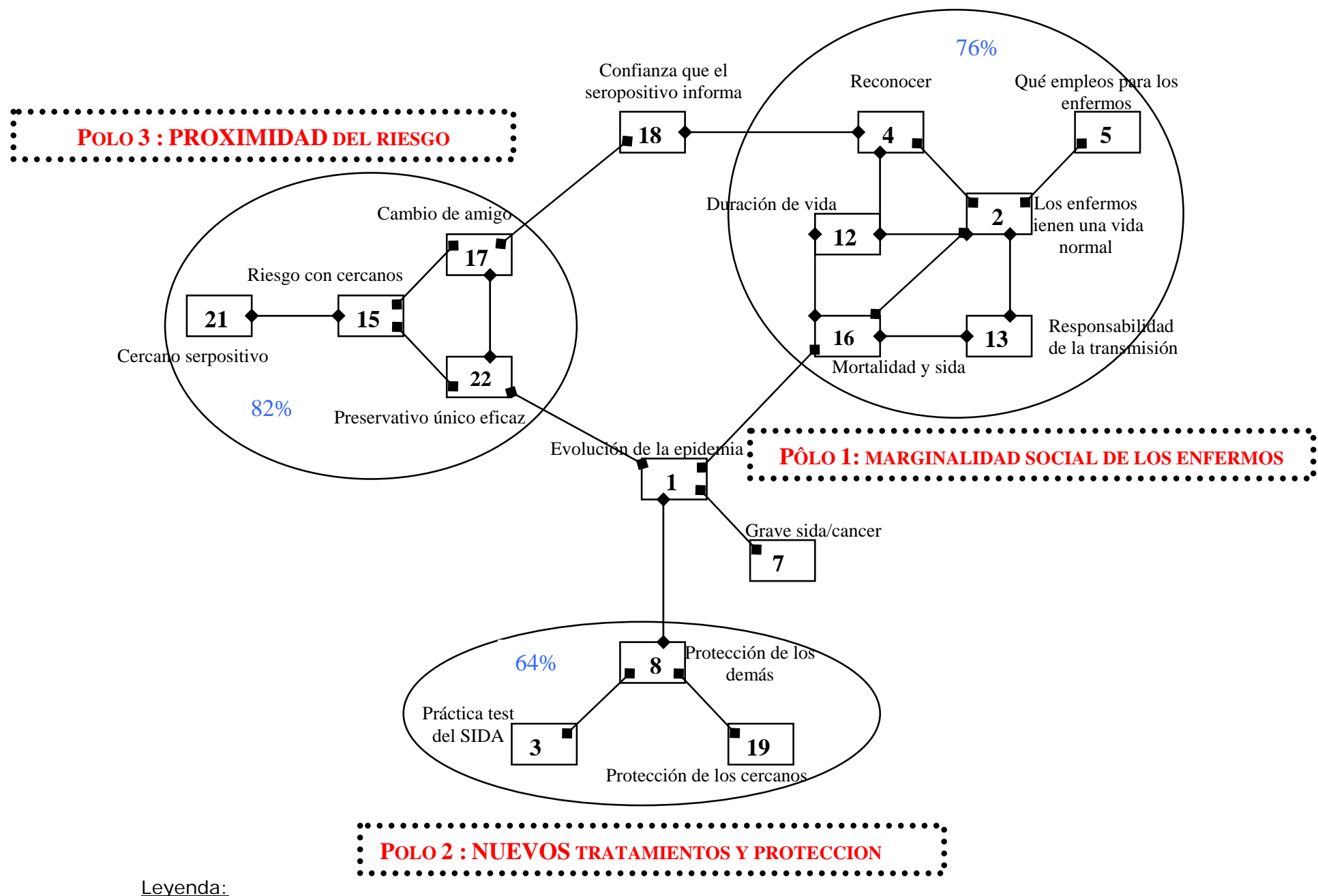

Leyenda:

Cada ítem está representado por su número inicial y un enunciado sintético de su contenido

$\longleftrightarrow$ Dependencia significativa y recíproca entre dos ítems

Los polos de ítems interdependientes

xx\% Resultado medio de consenso por área de representación

Figura 1. Las interdependencias binarias entre 16 ítems. Nota: La longitud de los lazos no es significativa

Lectura: Por ejemplo, respecto al tema del polo 3, la opinión relativa a "si un amigo toma riesgos, es posible hacerle cambiar de comportamiento" (17) está ligada (positivamente o negativamente) a la opinión respecto a "sólo el preservativo permite protegerse eficazmente del sida" (22). Decir que (no) se corren menos riesgos si se tienen relaciones sexuales con personas cercanas respecto a hacerlo con desconocidos (15) está directamente ligado por una parte, a las dos opiniones anteriores, por otra parte, al hecho de decir que (no) es posible que una persona cercana sea seropositiva y que no lo sepamos (21). Estas 4 opiniones, agrupadas en torno al ítem 15 forman el área de representaciones que hemos llamado "proximidad del riesgo".

Encontramos aquí las temáticas principales que definen tres áreas de opiniones: 
REDES- Revista hispana para el análisis de redes sociales

Vol. 18, \#1 Junio 2010

http://revista-redes. rediris.es

- Una primera área de representaciones, que llamamos "marginalidad bio-social de los enfermos", está formada por 6 ítems interconectados que hacen referencia al efecto de los nuevos tratamientos sobre la enfermedad (letal o crónica?) y sobre la situación médica y social de los enfermos. Este área asocia todos los ítems relativos a los enfermos $(12,2,5,4,18)$ que parecen concentrarse alrededor del ítem 2 "Gracias a la existencia de nuevos tratamientos, las personas que han declarado la enfermedad del sida pueden tener una vida normal".

Aquí distinguimos bien un área de representaciones centrada en el carácter letal o crónico de la enfermedad en relación con el reconocimiento de los enfermos (4 y 5) y la responsabilidad de la transmisión (13 y 18). El ítem relativo a la confianza que se tiene en las personas seropositivas de que informen a sus parejas liga este área de representaciones con:

- el área de la "cercanía del riesgo", que agrupa alrededor de la idea de que se corre menos riesgo con personas cercanas que con desconocidos (15) las opiniones relativas a lo que se dice en las relaciones de amistad sobre el sida y los riesgos asociados.

- Encontramos finalmente la temática estructurante del mantenimiento/declive de la protección desde la introducción de los nuevos tratamientos, formada por la pareja de ítems 19 "A causa de los nuevos tratamientos, algunas de vuestras personas cercanas se protegen menos que antes" y "a causa de los nuevos tratamientos, algunas personas se protegen menos que antes", a los que se añade el ítem 3 "el test de diagnóstico debe practicarse siempre después de una relación con una nueva pareja sexual".

Esta última área de representaciones está indirectamente ligado a los dos otros por un intermediario, el ítem 1 "La epidemia del sida afecta cada vez menos personas en Francia" al que se asocia el ítem 7 "El sida es una enfermedad más grave que el cáncer". No nos sorprende constatar que el ítem más general de la serie, que describe globalmente la evolución de la epidemia, sea el vínculo entre las tres áreas de representación distinguidas.

Finalmente, hemos establecido las puntuaciones medias de consenso por área (\% en el esquema), es decir que hemos calculado la media de las opiniones más citadas por ítem (es decir, la media de las puntuaciones de acuerdo - si es la más citada - o desacuerdo si es la más consensual - de cada ítem por área).

Constatamos que, efectivamente, las puntuaciones difieren entre ellas:

- Si el área de la marginalidad bio-social de los enfermos presenta una puntuación intermediaria con un consenso medio de 76\% (mínimo 66\%, ítem 2 - máximo $86 \%$ ítem 12),

- el área de la proximidad del riesgo se caracteriza por un fuerte consenso con una puntuación media de 82\% (min. 77\%, ítem 15 - máx. 09\%, ítem 21), 
REDES- Revista hispana para el análisis de redes sociales

Vol. 18, \#1 Junio 2010

http://revista-redes. rediris.es

- e inversamente, el área de la percepción de la protección por la introducción de nuevos tratamientos presenta una puntuación media con un consenso claramente menor con $64 \%$ ( $\min .56 \%$ ítem 8 - máx. 68\% ítems 3 y 19).

Podemos pensar razonablemente que estas diferencias entre las puntuaciones medias de consenso por área traducen los grados de incertidumbre de las variables. Las distintas áreas de representaciones relativas al sida no presentan el mismo grado de incertidumbre.

El área de la proximidad del riesgo es la que tiende más hacia la certidumbre (los jóvenes parecen tener la certeza de que el riesgo no esta muy lejos). A la inversa, los efectos que pueden tener los nuevos tratamientos en el comportamiento de protección están cargados de incertidumbre. (Podemos por otra parte suponer que la forma lógica del área, que deriva de una hipótesis de imputación causal, no sea ajena a esta fuerte incertidumbre: ya no se trata solo de opinar a partir de una afirmación simple como "el sida puede afectar a tus personas cercanas" sino de evaluar la relación entre la introducción de los nuevos tratamientos y la modificación o no de los comportamientos de protección, es decir, las consecuencias posibles de un hecho sobre otro). Finalmente, las incertidumbres subsisten en cuanto a la marginalidad bio-social de los enfermos, aunque una opinión más fuerte ponga de acuerdo, en promedio, a tres actores de cada cuatro.

\section{Algunas lecciones...}

Las incertidumbres respecto al sida subsisten ampliamente entre ciertos representantes de la generación post-sida, y, particularmente en lo que se refiere a la percepción de los efectos de la introducción de los nuevos tratamientos en los comportamientos de prevención. Este área presenta una forma lógica específica que puede contribuir a explicar el fuerte grado de incertidumbre que la caracteriza, sin embargo, realza una distinción inquietante que parece marcar la generación: si bien una minoría estima que la llegada de los nuevos tratamientos no ha modificado los comportamientos de protección de "ciertas personas", más de 2 de cada 3 encuestados consideran que sus "personas cercanas" se protegen tanto como antes. Dicho de otro modo, estos encuestados tienen una visión particularmente optimista, por no decir ingenua del mantenimiento de los comportamientos de protección desde la introducción de las tri-terapias cuando se trata de sus personas cercanas. Esta interpretación es confirmada en el área de la proximidad del riesgo: mientras que 9 encuestados de cada 10 admiten que podrían tener una persona cercana que fuera seropositiva sin saberlo, 1 de cada 4 estima que se corre menos riesgo si se tienen relaciones sexuales con personas cercanas que con desconocidos. 
REDES- Revista hispana para el análisis de redes sociales

Vol. 18, \#1 Junio 2010

http://revista-redes. rediris.es

Si tenemos la costumbre de leer que los comportamientos rara vez van asociados a las opiniones, es menos frecuente admitir que las ideas no estén ligadas entre ellas. $Y$ sin embargo he aquí precisamente lo que revelan los análisis de la estructura de opiniones que hemos llevado a cabo: hemos revelado una estructura de opiniones, sin embargo esta estructura está innegablemente segmentada. Si decir que, "gracias a los nuevos tratamientos se muere menos de sida" está ligado al hecho de decir que "ya no se puede reconocer a las personas afectadas", esas opiniones no tienen nada que ver con la idea de que se corre menos riesgo con personas cercanas que con desconocidos, ni con la percepción de los efectos que los nuevos tratamientos han podido tener sobre los comportamientos de protección.

Esta segmentación del área de las representaciones relativas al sida implica, en términos de prevención, que se deben enviar mensajes específicos para cada área. Las políticas de prevención no se pueden apoyar en una lógica de tipo deductivo del estilo "si ya sé que no se puede reconocer a las personas afectadas de sida, entonces puedo tener una persona cercana seropositiva sin saberlo, por lo tanto no corro menos riesgos si tengo relaciones sexuales con una persona cercana que con un desconocido". La prevención debe tener en cuenta la desconexión de las áreas (que se traduce por una ausencia de relación lógica entre dichas proposiciones) de la misma manera que debe tener en cuenta la falta de relación entre opiniones y comportamientos.

\section{La convergencia de las opiniones en las díadas íntimas}

Las opiniones inciertas relativas al sida expresadas por dos actores que interactúan entre ellos convergen más o menos en función de un aspecto específico de la relación: al contar el número de opiniones divergentes (uno está de acuerdo y el otro no) emitidas simultáneamente por dos interlocutores sobre una serie de 16 ítems relativos al sida, podemos distinguir dos perfiles diádicos: las díadas "convergentes" que presentan como máximo 4 opiniones divergentes y las díadas "divergentes" cuando presentan al menos 5 opiniones divergentes.

Se pueden formular diferentes hipótesis sobre esta problemática de convergencia. Se podría pensar que los miembros de una pareja tienen tendencia a expresar opiniones más cercanas que dos amigos que discuten entre ellos, o que dos hombres tendrían opiniones más convergentes que una díada mixta. Por ejemplo, nos esperábamos que las opiniones expresadas conjuntamente por los miembros de una pareja relativas a las 16 proposiciones fuesen más a menudo parecidas que las opiniones emitidas por amigos. Los resultados no muestran en absoluto esta tendencia: la pareja constituye precisamente la relación con las opiniones menos frecuentemente convergentes en cuanto al sida se refiere ( $56 \%$ de las díadas de parejas son convergentes contra $67 \%$ de 
REDES- Revista hispana para el análisis de redes sociales

Vol. 18, \#1 Junio 2010

http://revista-redes. rediris.es

las díadas de amigos), y dos hombres estarán menos a menudo de acuerdo entre ellos ( $42 \%$ de las díadas masculinas son convergentes) que un hombre y una mujer (56\% de las díadas mixtas convergen), siendo la relación femenina la más convergente de todas (72\% de díadas convergentes entre las díadas femeninas).

Pero ninguna característica relacional - tipo de relación, género de la díada...- logra explicar mejor la convergencia de opiniones entre dos actores que su intimidad en el sentido de la confidencia. Cuando se trata de expresarse respecto al sida, los actores que están más a menudo de acuerdo son los que tienen la costumbre de hablar entre ellos de cuestiones personales, tanto si son de mismo sexo como si no y cualquiera que sea el tipo de relaciones que mantengan.

No se comparten sistemáticamente las opiniones de los interlocutores, ya sean amigos, padres o pareja, pero podemos pensar que hay una lógica relacional en la formación de una opinión común entre dos actores. Si esta lógica está asociada a la confidencia cuando se trata del sida, se puede pensar que el criterio relacional de la convergencia varía en función del tema abordado: por ejemplo, si se trata de un riesgo de salud "espontáneo" como el cáncer de mama, la autoridad científica en la relación podría contribuir más fuertemente a la formación de un consenso que la intimidad de los interlocutores (se trata de una hipótesis).

Por otra parte, este resultado nos invita a suponer que un mensaje será tanto más perenne que será compartido, más que vehiculado, entre dos actores que tienen costumbre de discutir juntos de cuestiones personales.

Esta constatación impone un verdadero desafío en términos de prevención: ¿qué procedimiento será susceptible de provocar discusiones y conversaciones "íntimas" que garanticen que se compartan las "buenas opiniones" relativas al conocimiento de la enfermedad y a los riesgos de transmisión? Esta cuestión deriva no una sino tres consecuencias de cara a la prevención:

- Habría que provocar debate, los jóvenes deben hablar del sida.

- Sería necesario que dichas conversaciones tengan lugar entre personas que tienen a costumbre de abordar cuestiones personales juntas - los jóvenes deben hablar de sida con personas que les resulten íntimas para que las opiniones que comparten adquieran un valor, una fuerza, una persistencia.

- Pero ¿cómo asegurarse de que la producción normativa de dichas conversaciones íntimas será "buena" para la prevención? - los jóvenes deben compartir las "buenas ideas" que permitan una buena prevención del sida. 
REDES- Revista hispana para el análisis de redes sociales

Vol. 18, \#1 Junio 2010

http://revista-redes. rediris.es

La idea parece descabellada, sin embargo, a la luz de este estudio, un mensaje de prevención no tendrá nunca tanto valor como por el etiquetado ${ }^{2}$, que le pueda conferir un intercambio personal íntimo.

\section{La estabilización de las opiniones en las estructuras sociométricas cerradas}

Abordemos un último problema discutible en el marco de este estudio: ¿cambian de opinión los actores cuando interactúan sucesivamente con dos personas diferentes sobre las mismas cuestiones relativas al sida? Por ejemplo, un estudiante que da su opinión sobre las mismas proposiciones relativas al sida en un primer momento con una amiga de su elección, y quince días después con su padre, ¿expresará opiniones diferentes o sistemáticamente similares para los 16 ítems? Es fuerza reconocer la respuesta afirmativa a la primera proposición. El estudio nos muestra que un tercio de los actores cambia al menos un cuarto de sus opiniones. Por lo tanto podemos distinguir una categoría de actores más bien estables (menos de 3 opiniones versátiles) y una categoría de actores más bien versátiles (que cambian de opinión al menos 3 veces respecto a las 16 proposiciones entre los dos momentos en que se pasó el cuestionario).

Si se descartan inmediatamente las hipótesis individualistas de tipo "ciertas personas tienen tendencia a la versatilidad porque están menos seguras de sí mismas" ¿se puede explicar la tendencia a la estabilidad o a la versatilidad del actor de acuerdo con la estructura relacional de discusión en la que se encuentra? La "estructura relacional de discusión" designa la configuración de la tríada formada por el estudiante al escoger dichos interlocutores y varía en función de dos informaciones relativas a la percepción que el estudiante tiene de la estructura relacional:

- la clausura/apertura de la tríada: la tríada se llama cerrada si el estudiante ha declarado que sus interlocutores se conocen o disjunta si afirma que no se conocen,

- la tipología de las pertenencias de los actores de la tríada, que se puede definir de la manera siguiente:

a) La pertenencia única: los interlocutores están insertos en un mismo grupo al que pertenecen:

\footnotetext{
2 Por "etiquetaje" de las opiniones, designamos un marcaje relacional que será más o menos fuerte en función de ciertas características de la relación asociadas a la opinión en juego. En concreto, hemos mostrado que las opiniones relativas al sida están más fuertemente marcadas cuando son compartidas en relaciones íntimas que cuando se producen en relaciones distantes. NDT, respecto al concepto de "etiquetaje" véase el artículo de Alexis Ferrand en la revista REDES 2006, Vol. 10, \#2.
} 
REDES- Revista hispana para el análisis de redes sociales

Vol. 18, \#1 Junio 2010

http://revista-redes. rediris.es

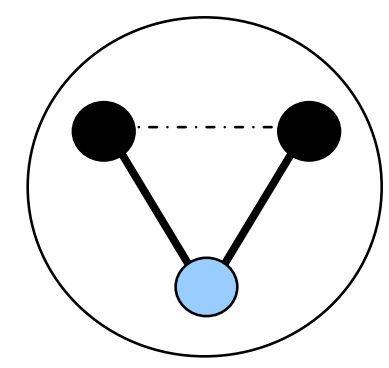

b) las pertenencias duales: los interlocutores están insertos en dos grupos separados a los que ego pertenece:

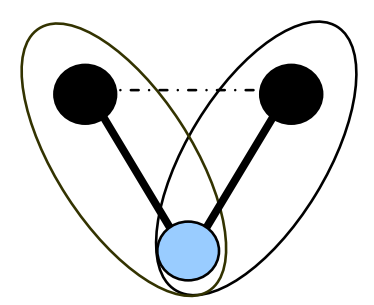

c) Ia pertenencia aislada: el interlocutor de un cuestionario como mucho está inserto en un grupo al que pertenece ego
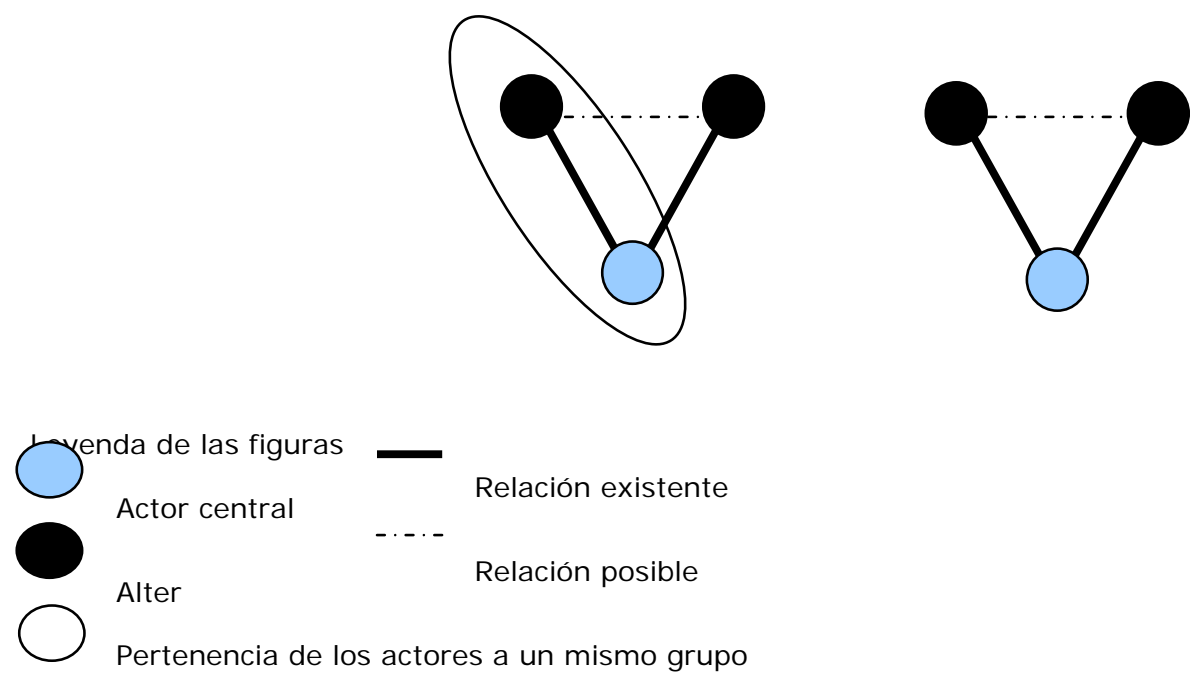

Figura 2. Topología de las pertenencias de los actores de la tríada.

Nota: las líneas representan las relaciones entre actores que constituyen la tríada, los círculos simbolizan el hecho de que los actores pertenecen o no un mismo grupo.

Este interrogante explora posibilidades como las siguientes:

- cuando dos interlocutores sucesivos de un estudiante se conocen, podemos emitir la hipótesis de que sus opiniones se parecen particularmente (según la idea de que "quien se parece se reúne" ${ }^{3}$, bien sea a la inversa, suponiendo que, dado que se frecuentan, desarrollan o comparten ideas "comunes"), y que dichas tríadas cerradas presentan por eso un contexto de opiniones particularmente homogéneo. Dicho de otro modo, el estudiante estaría confrontado, en esta configuración, a

\footnotetext{
${ }^{3}$ NDT : dicho francés similar a « cada oveja con su pareja »
} 
REDES- Revista hispana para el análisis de redes sociales

Vol. 18, \#1 Junio 2010

http://revista-redes. rediris.es

opiniones particularmente similares entre los dos cuestionarios y tendería por lo tanto a una fuerte estabilidad en sus respuestas. Tendríamos entonces un efecto de la estructura relacional de la discusión - estructura cerrada - en la estabilidad de las opiniones - "cambio poco frecuente de las respuestas del estudiante entre los dos cuestionarios". Sin embargo, si bien observamos una estabilidad específica de las opiniones en las tríadas cerradas, la hipótesis inicial según la que los interlocutores que se conocen tienen opiniones particularmente similares entre ellas ha sido invalidada. Esta constatación invita a suponer que ciertos grupos podrían estar formados por personas que "hacen cosas juntos" pero que no conversan particularmente, o al menos que no lo hacen sobre temas como el sida.

Así pues hemos explorado otra hipótesis para explicar esta estabilidad de las opiniones:

- un estudiante de sociología que habla sucesivamente con un(a) amigo(a) y después con su pareja o con uno de sus padres, o un primo, u otro de sus amigos, podría tender a expresar opiniones particularmente estables porque percibe que sus dos interlocutores se conocen.

La constatación principal es efectivamente la siguiente: un actor tiende a expresar más a menudo opiniones idénticas cuando sus interlocutores se conocen, pero tanto más cuando éstos pertenecen (en su opinión) al mismo grupo relacional del cual él mismo forma parte. A la inversa, los actores más versátiles son aquellos que dicen que sus interlocutores pertenecen a grupos diferentes o a ningún grupo que ellos sepan. Frente a esta constatación, la primera hipótesis presentada -según la cual, a modo de recordatorio, los interlocutores que pertenecen a un mismo grupo tienen ideas más similares que los que no forman parte de un mismo grupo relacional, y que es ésta homogeneidad cognitiva del contexto relacional la que explica la estabilidad de las opiniones de los actores- no se sostiene, dado que los interlocutores pertenecientes a un mismo grupo no tienen opiniones más similares que los demás. Esta hipótesis se ve por lo tanto descartada a favor de la lógica puramente estructural de la estabilidad: un actor que percibe la clausura del contexto relacional en el que se expresa vacilará antes de emitir dos opiniones diferentes para no correr el riesgo de que sus interlocutores se den cuenta y hablen entre ellos de su versatilidad. Sin embargo un actor que percibe una estructura relacional disjunta - en la que los interlocutores no podrían volver a hablar entre ellos de la cuestión - será más libre de expresar opiniones contradictorias en función de la persona con quien interactúa. Esta lógica estructural de estabilización de las opiniones en las tríadas cerradas puede ser designada "presión social a la coherencia cognitiva".

Hemos por lo tanto identificado un factor propiamente estructural - la percepción de un contexto relacional disjunto - en un proceso de formación de opiniones inciertas - la versatilidad de las opiniones. 
REDES- Revista hispana para el análisis de redes sociales

Vol. 18, \#1 Junio 2010

http://revista-redes. rediris.es

Pero ¿desacredita realmente ser versátil? ¿En qué medida un actor está sometido a dicha presión? ¿Será la presión diferente en función del tipo de grupo a que pertenece el actor - familia vs. amistad, por ejemplo, o confidente vs. festiva? Esta presión social a la coherencia cognitiva ¿es de misma intensidad cualquiera que sea el tema discutido? Dicho de otro modo, ¿asumirían los actores aún más o menos una versatilidad política que una versatilidad sobre una cuestión de salud? ¿Se permitirá con más facilidad un actor cambiar de opinión respecto a un contenido de conocimiento que respecto a un juicio? Por ejemplo, podría ser que yo admitiera más fácilmente tener opiniones contradictorias sobre un ítem tal como "la epidemia del sida afecta cada vez a menos personas en Francia" que sobre una proposición del tipo "los enfermos de sida pueden ocupar cualquier tipo de empleo" porque la primera no me implica más que a nivel de "lo que sé o creo de la epidemia", mientras que la segunda me implica respecto a la manera en que juzgo la situación de los enfermos respecto a otros y, más particularmente, su "posición en la vida social".

\section{Conclusión}

Hemos establecido que la producción de las opiniones en la tríada resulta de la activación cruzada:

- del etiquetaje relacional de las opiniones en la interacción, respecto al sida, la fuerza de este "etiquetaje" depende de la intimidad de la relación,

- de la percepción de la estructura sociométrica en la que el actor está inserto con sus interlocutores: estabilización de las opiniones cuando el actor percibe la clausura de la tríada.

De un punto de vista subjetivo, podemos enunciar estos procesos relacionales del modo siguiente:

- La convergencia diádica: "si hablo del sida con una persona íntima, tendré tendencia a ponerme de acuerdo con ella, y esta opinión compartida tendrá su importancia, mientras que discuto con una persona distante, poco importa que no estemos de acuerdo. Así pues, si mis interlocutores tienden a tener opiniones diferentes, y que sólo una de estas relaciones es íntima, no compartiré mas que la opinión formada en esta relación, sin embargo, si mis dos interlocutores sucesivos son íntimos y tienen opiniones diferentes, tenderá a compartir la opinión de cada uno de ellos, y por lo tanto, a tener dos opiniones diferentes".

- La estabilización de las opiniones en las tríadas de pertenencia única: "Si percibo que mis interlocutores son susceptibles de hablar entre ellos de las opiniones emitidas en contextos separados, tendré tendencia a expresar la misma opinión frente a cada uno de ellos. Por lo tanto, si mis interlocutores tienden a expresar opiniones diferentes, mantendré una opinión estable si ambos pertenecen al mismo grupo que yo, y me preocuparé menos por ello si no percibo dicha relación entre nosotros".

Nos preguntamos entonces qué sucede:

- cuando ego es íntimo con cada uno de sus interlocutores, tendiendo por ello a la convergencia en cada una de las relaciones y a posiciones sucesivas potencialmente contradictorias, 
REDES- Revista hispana para el análisis de redes sociales

Vol. 18, \#1 Junio 2010

http://revista-redes. rediris.es

y a la vez,

- sus interlocutores están insertos en el mismo grupo que él, invitándole por el contrario a la estabilización de sus opiniones.

La exploración de esta situación relacional que activa la competencia entre las lógicas de producción de las opiniones que hemos identificado ha mostrado que esta competencia queda irresuelta: no hemos podido concluir con la supremacía de una lógica sobre la otra. 